\title{
A Case of a 39-year-old Immunocompromised Filipino Male with Non-Healing Wound of the Right Lower Leg
}

\author{
Janice Jill K. Lao, Tennille S. Tan, Alex P. Bello, Malen Uichangco-Bravo, \\ Emily Ruiz-Jacinto, Allan D. Corpuz and Agnes D. Mejia \\ Department of Medicine, College of Medicine and Philippine General Hospital, University of the Philippines Manila \\ ${ }^{2}$ Center for Natural Sciences and Environmental Research, De la Salle University, Taft Avenue, Manila
}

\begin{abstract}
This is a case of a 39-year-old Filipino male with systemic lupus erythematosus (SLE) diagnosed in 2006, presenting with a 3month history of non-healing wound on his right lower leg. This paper will discuss the etiologies of a non-healing wound and present an algorithm to guide the approach to diagnosis and management.
\end{abstract}

Key Words: lupus, non-healing wound, Filipino

\section{Case Presentation}

A 39-year-old Filipino male was diagnosed with SLE in 2006 (3 years prior to admission), presenting with hypertension, on-and-off bipedal edema, headache, malar and discoid rash, oral ulcers, arthralgia, myalgia, bilateral flank pains, and frothy urine. His Anti-nuclear antibodies (ANA) and anti-double-stranded DNA (anti-dsDNA) were positive. He also had thrombocytopenia, and acquired coagulopathy. He was started on steroids with good compliance.

In 2007 (2 years prior to admission), he was hospitalized for a midbrain cerebrovascular bleed and was discharged with right-sided hemiparesis. Anti-phospholipid antibody syndrome (APAS) was ruled out. Later that year, he was admitted for pleural effusion and was started on anti-Koch's therapy.

In February 2008 (1 year prior to admission), he consented to a kidney biopsy which showed diffuse segmental proliferative and sclerosing lupus nephritis with $18 \%$ global sclerosis, $36 \%$ segmental sclerosis, and $18 \%$ fibrous crescents (Class IV).

In July 2008 (5 months prior to admission), he was started on Cyclophosphamide pulse therapy due to persistent heavy proteinuria. Mycophenolate mofetil was added on his $2^{\text {nd }}$ cycle of cyclophosphamide pulse therapy.

Corresponding author: Agnes D. Mejia, MD

Department of Medicine

Philippine General Hospital

University of the Philippines Manila

Taft Avenue, Ermita, Manila 1000 Philippines

Telephone: +6325548400 local 2200/2206

Telefax: +632 5264372

Email: agnesmejiamd@gmail.com
After his $3^{\text {rd }}$ cycle of Cyclophosphamide in September 2008, he developed swelling in his right lower leg with erythema, warmth, and tenderness. The medial side of the leg eventually became hyperpigmented, with erythema, edema, and ulcerations draining purulent discharge. He had fever and chills. For the next 3 months, he was repeatedly admitted and treated as a case of chronic venous insufficiency and cellulitis. He received several antibiotics like Oxacillin, Ciprofloxacin, Vancomycin, Clindamycin and Ampicillin-Sulbactam. Inspite of these drugs, there was minimal improvement.

On follow-up in November 2008 (1 month prior to admission), there were new ulcerations noted at the lateral malleolus with satellite lesions at the medial aspect of the right lower leg. He took oral Ciprofloxacin and Clindamycin for a week. However, due to worsening of the wound, he was admitted at the University of the Philippines-Philippine General Hospital (UP-PGH).

On admission, the patient had normal vital signs. Pertinent physical exam findings included pale palpebral conjunctivae, edematous right lower leg with a $3 \times 2 \mathrm{~cm}$ ulcer on the medial side and a $1 \times 2 \mathrm{~cm}$ ulcer on the right ankle, with yellowish to whitish discharge (Figure 1). Leg edema improved with judicious compressive bandaging and leg elevation. However, due to the persistence of fever, despite negative cultures, antibiotics were empirically shifted to piperacillin-tazobactam. The patient was referred to the Section of Dermatology. Multiple petechiae and purpura on the upper extremities were consistent with cutaneous lupus erythematotus (Figure 2). The multiple raw-based ulcers in the right lower leg draining purulent discharge with well-defined border (Figure 3) showed suppurative and granulomatous infiltrates still consistent with chronic venous insufficiency with secondary bacterial infection.

Surgical debridement was done. Multiple draining sinuses in the medial aspect of the right leg were seen. Tissue samples taken yielded Methicillin-resistant Staphylococcus epidermidis (MRSE) sensitive to vancomycin. Despite debridement, the wound continued to drain purulent and bloody discharge, hence, piperacillintazobactam was empirically shifted to imipenem and vancomycin. Purulent discharge decreased but with poor 
granulation. Another wound C/S yielded acid-fast bacilli and growth of Mycobacterium sp. on culture. Doxycyline, Streptomycin, Ethambutol, and Ofloxacin were started.

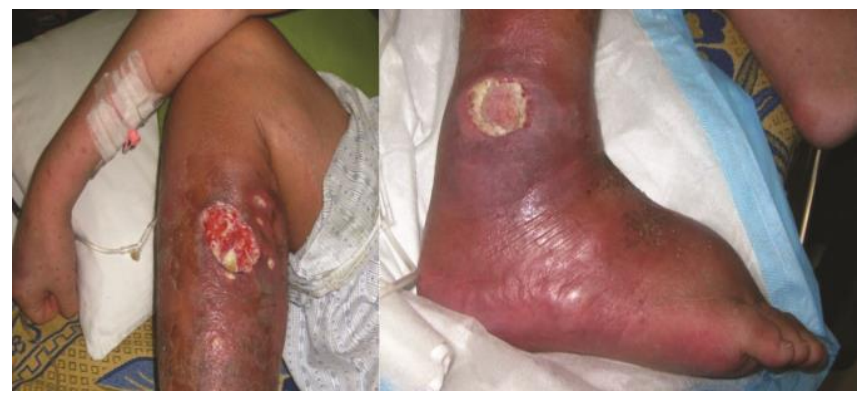

Figure 1. Right lower leg with ulcers on the medial side and ankle.

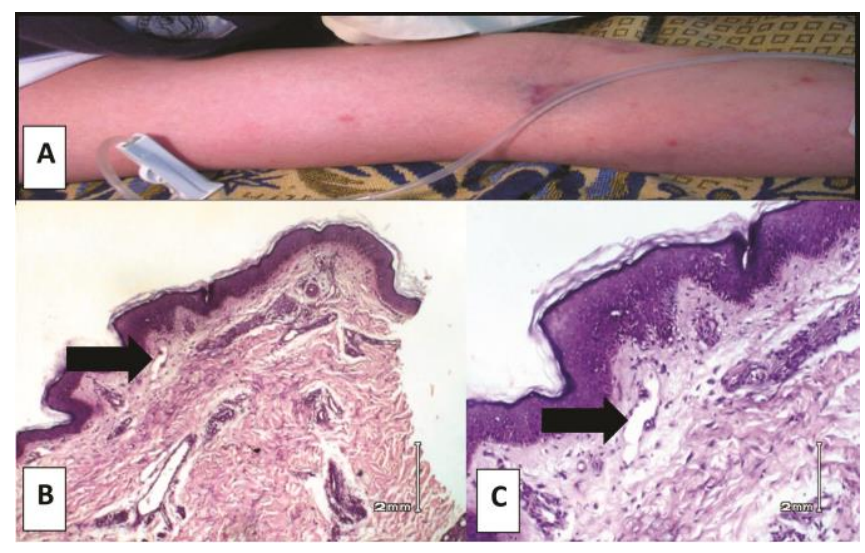

Figure 2. Upper arm biopsy. A. Patient's right arm showing multiple erythematous macules; B. Low power field showing dilated superficial vessels (arrow) and sparse superficial perivascular infiltrates; C. High power field showing dilated blood vessels (arrow) and mild vacuolar interface.

After surgery, the patient also developed nosocomial pneumonia. Sputum culture revealed Acinetobacter baumani and ampicillin-sulbactam was added. Fungal pneumonia was also entertained because of worsening oral thrush. This was treated with fluconazole. He eventually succumbed to septic shock. Post mortem wound culture revealed Mycobacterium tuberculosis.

\section{Discussion}

Chronic or non-healing wound is defined as a wound that fails to heal after 4-8 weeks despite treatment. ${ }^{1}$ The approach to a non-healing wound despite seemingly appropriate management is to refocus on complete history and physical exam and to consider performing a wound biopsy early. Failure to heal is often a result of misdiagnosis. Table 1 shows several local and systemic factors that must be considered in non-healing wounds while Table 2 states indications for wound biopsy. ${ }^{2,3}$ Biopsy of a wound base and its margins is usually advocated for wounds that have been present for longer than three months and wounds that have not responded to standard therapy. ${ }^{2}$

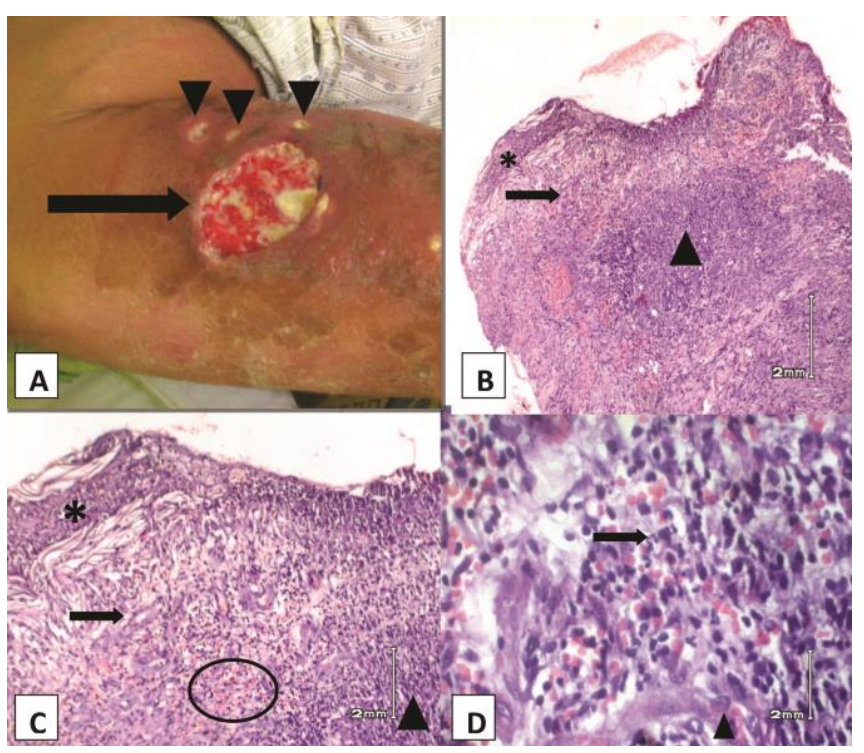

Figure 3. Leg ulcer biopsy. A. Necrotic ulcer with undermined edges (arrow) on the right medial lower leg with small, surrounding satellite ulcers (arrowheads), purulent discharge; B. Low power field: Loss of full thickness of the epidermis $\left(^{*}\right)$ with clusters of thick walled blood vessels (arrow) and dense granulomatous infiltrate in the dermis (arrowhead); C. High power field: Loss of full thickness of the epidermis $\left({ }^{*}\right)$ with clusters of thick walled blood vessels (arrow) and dense granulomatous infiltrate in the dermis (arrowhead) with note of extravasated erythrocytes (circle) in the dermis; D. Oil immersion field: Mixed infiltrate of neutrophils (arrow), histiocytes, scattered plasma cells (arrowhead) in the dermis.

Table 1. Causes of impaired wound healing

\begin{tabular}{lll}
\hline \multicolumn{1}{c}{ Local Factors } & \multicolumn{1}{c}{ Systemic Factors } \\
\hline - Repeated external trauma & - Arterial insufficiency \\
because of inappropriate & - Venous insufficiency \\
offloading & - Systemic conditions (collagen \\
- Foot deformity causing & vascular disease, sickle cell \\
abnormal pressure areas & disease, hemoglobinopathies, \\
- Uncontrolled edema & uremia, diabetes, jaundice) \\
- Injury from use of toxic substances & - Immunosuppresive drugs \\
- Inappropriate measures for & (systemic corticosteroids, \\
- drainage control & anticancer drugs, nsaids) \\
- Tnappropriate infection control & - Immunosuppresive diseases (hiv) \\
- Hematoma formation & - Local or systemic malignancy \\
- Undebrided wound & - Exposure to radiation \\
- Poor blood supply & - Malnutrition \\
- Poor surgical apposition & - Old age and general immobility \\
- Wound dehiscence & - Obesity \\
- Presence of foreign body and & - Deficiency of vitamins and trace \\
foreign body reactions & elements \\
\hline
\end{tabular}


Table 2. Indications for wound biopsy

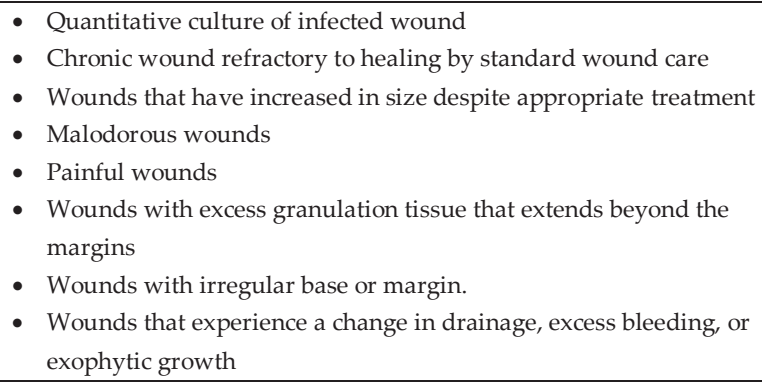

- Chronic wound refractory to healing by standard wound care

- Wounds that have increased in size despite appropriate treatment

- Malodorous wounds

- Painful wounds

- Wounds with excess granulation tissue that extends beyond the margins

- Wounds with irregular base or margin.

- Wounds that experience a change in drainage, excess bleeding, or exophytic growth

Our patient had uncontrolled leg edema, vascular insufficiency, immuno-compromised state, and was on immunuosuppressive drugs, all of which contributed to poor wound healing. Judicious medical management of the leg's venous insufficiency, with bandaging and leg elevation, resulted in notable improvement in his leg. Given the associated signs of local inflammation and systemic signs like fever and tender inguinal nodes, secondary infection of the wound was highly entertained.

Immunocompromised patients are susceptible to infections caused by a variety of microbial pathogens, $20 \%$ of which manifest as skin infections. Of these, more than two thirds are primary rather than secondary metastatic infections. Cutaneous manifestations are often accompanied by fever, defined as an isolated temperature of $38.3^{\circ} \mathrm{C}$ $\left(101^{\circ} \mathrm{F}\right)$ that cannot be attributed to exogenous causes, such as blood products, or a temperature above $38^{\circ} \mathrm{C}\left(100.4^{\circ} \mathrm{F}\right)$ that persists for more than 1 hour. Etiology is usually dependent on the defective mechanism in the immune system. ${ }^{4}$ In our patient, we inferred that this was due to neutrophilic dysfunction and cell-mediated defect on the background of SLE and steroid use.5,6

Cutaneous bacterial infections in immunocompromised hosts can range from the more common gram positive (Staphylococcus/Streptococcus), gram negative (Pseudomonas) or anaerobic organisms (Clostridium), to the more unusual Actinomycosis and Mycobacterium as shown in Tables 3 and 4. ${ }^{4}$ In our patient, the initial cultures were always negative suggesting that the initial ulcer might really be a stasis ulcer, or that the initial antibiotics were effective, or that the diagnostic measures done where not compatible with the etiologic organism. The suppurative infiltrates on biopsy did support a bacterial infectious etiology. Wound culture yielded methicillin-resistant Staphylococcus epidermidis (MRSE). While MRSE can be due to contamination, it was considered a pathogen in our patient, given his immunocompromised state. Culture-guided treatment eventually yielded improvement of wound symptoms but only temporarily, prompting new cultures to be done, including AFB staining.
Table 3. Skin pathogens in patients with neutrophilic dysfunction.

\begin{tabular}{ll}
\hline \multicolumn{1}{c}{ Bacteria } & \multicolumn{1}{c}{ Fungi } \\
\hline Pseudomonas aeruginosa & Aspergillus species \\
Stenotrophomonas maltophiliaa & Candida species \\
Aeromonas hydrophila & Trichosporon species \\
Enterobacteriaceae & Fusarium species \\
Viridans streptococci & Mucorales species \\
Staphylococcus aureus & \\
Staphylococcus epidermidis & \\
Corynebacterium jeikeium & \\
Bacillus species & \\
Clostridium species & \\
Bacteroides fragilis & \\
\hline
\end{tabular}

Table 4. Skin pathogens in patients with cell-mediated deficiency

\begin{tabular}{lcc}
\hline \multicolumn{1}{c}{ Bacteria } & Fungi & Viruses \\
\hline Mycobacteria species & Histoplasma & Herpes simplex \\
Nocardia species & capsulatum & virus \\
Salmonella & Coccidioides & Varicella-zoster \\
species & immitis & virus \\
Listeria & Blastomyces & Cytomegalovirus \\
monocytogenes & dermatitidis & Epstein-Barr \\
Legionella & Penicillium & Virus \\
species & marneffei & \\
Bartonella & Cryptococcus & \\
species & neoformans & \\
Brucella species & Aspergillus & \\
& species & \\
& Candida & \\
\end{tabular}

Other Mycobacteria to be considered are Mycobacterium leprae and Non-tuberculous Mycobacteria (NTM). A study in India, on the clinico-epidemiolgic profile of non-healing wounds in one of their hospitals revealed leprosy as the leading cause, followed by diabetes, trauma, and venous ulcers. ${ }^{7}$ A review of the UP-PGH Section of Dermatology biopsy census for 2010 revealed Hansen's disease or leprosy as the most common diagnosis for questionable skin lesions. ${ }^{8}$

Leprosy is caused by Mycobacterium leprae, an acid-fast bacillus, indistinguishable microscopically from other Mycobacteria, and ideally detected in tissue sections by a modified Fite stain. ${ }^{9}$ The clinical presentation of leprosy, specifically the skin lesions, is diverse and is due to the host's capacity to generate cell-mediated immunity. ${ }^{10}$ In patients with deficient cell-mediated immunity, the lepromatous type of leprosy (LL) is favored, which is usually associated with numerous, poorly demarcated, raised or nodularlesions on all parts of the body. Biopsies reveal sheets of foamy macrophages in the dermis containing very large numbers of bacilli and microcolonies. ${ }^{10}$ These, however, were not seen in our patient's biopsy. Furthermore, ulcerations in leprosy are usually associated with the tuberculoid type (TL) and are usually seen at the foot's plantar aspect due to neuropathy. ${ }^{9,11}$ There is another type of ulceration in leprosy termed non-neuropathic 
nonspecific ulcer which is similar to stasis ulcers, except that this type of ulceration is usually seen in elderly women previously treated for lepromatous leprosy and is usually AFB negative. ${ }^{11}$

Another more common acid-fast organism especially in the local setting is Mycobacterium tuberculosis. Our patient was previously treated for pulmonary tuberculosis (TB), hence, reactivation due to immunosuppression is highly likely. Cutaneous TB infections are rare, accounting for only less than $0.1 \%$ of individuals seen in dermatology clinics in high prevalence areas like India or China. ${ }^{12}$ A review of the biopsy census of the UP-PGH Section of Dermatology from 2004 to 2010 revealed only 6 cases of TB of the skin and soft tissue infection $(<0.01 \%)$ in adult patients. Cutaneous TB can present as primary infection due to direct inoculation, abscesses and chronic ulcers, scrofuloderma, lupus vulgaris (a smoldering disease with nodules, plaques, and fissures), miliary lesions, and erythema nodosum. ${ }^{9}$

Both M. leprae and M. tuberculosis form granulomas on biopsy, consistent with those seen in our patient's biopsy. Infection with NTM other than M. leprae should also be considered since these organisms are prevalent in the environment and present increasingly as a cause of skin infections among immunocompromised individuals. ${ }^{13,14}$ Like the above two Mycobacteria, NTM also cause slowly developing chronic skin infections. ${ }^{14}$ Almost all NTM species have been noted in cutaneous diseases and these include - but are not limited to - M. marinum (swimming pool and fish tank granuloma), M. ulcerans (Buruli ulcer), $M$. fortuitum, M. kansasii, and M. avium intracellulare (MAI). ${ }^{15} \mathrm{M}$. marinum, considered the most common among the NTM ${ }^{15}$ usually presents as a small violet nodule or pustule at a site of minor trauma which then evolve to form a crusted ulcer or small abscess. It often heals spontaneously. ${ }^{2}$ M. ulcerans may cause an itching or painless erythematous nodule on arms or legs, which then breaks down to form a shallow ulcer of variable size..$^{2,14} \mathrm{M}$. fortuitum usually presents as nodular lesions in immunosuppressed individuals and are rapidly growing Mycobacteria known to be resistant to most antituberculous drugs. ${ }^{2}$ M. kansasii presents with localised primary cutaneous lesions among immunocompetent individuals, but presents with disseminated or lung lesions in those who are immunocompromised. It may resemble cellulitis or sporotrichosis. ${ }^{14}$ MAI infection may involve the skin primarily through posttraumatic inoculation, ${ }^{14,16,17}$ secondarily as a manifestation of disseminated MAI, or by direct extension as a complication of cervical lymphadenitis. ${ }^{16}$ The skin lesions are characterized by ulceration, abscess, or erythematous plaque with little or no lymph node involvement. ${ }^{14,17}$

Histopathologic patterns of NTM may include nonspecific dermal or subcutaneous histiocytic inflammation, abscesses, tuberculoid or sarcoidal granulomas, and rheumatoid-like nodules. ${ }^{13,15}$ Tissue cultures or polymerase chain reaction (PCR) analysis of tissue samples are needed to establish the diagnosis. ${ }^{13-15}$ Culture is usually favoured over PCR so drug sensitivity can be determined. ${ }^{15}$ Disseminated forms are rare even in immunocompromised individuals and most are sensitive to Clarithromycin. ${ }^{13}$ Buruli ulcer, due to M. ulcerans, needs wide excision and debridement. ${ }^{14}$ Azithromycin is usually recommended for MAI. ${ }^{14,17}$

The Mycobacterium genus is not the only group of organisms that shows acid-fast activity. Other acid-fast organisms include Nocardia, Cryptosporidium paroum, Isospora belli, Cyclospora cayatenensis, and endospore-forming bacteria. ${ }^{18}$ Other than Nocardia and endospore-forming bacteria, there is no report yet of skin ulcerations or dermatologic manifestations for the other 3 organisms. Endospore-forming bacteria often yield positive Gram stain. Nocardia is an uncommon pathogen that affects both immunosuppressed and immunocompetent patients. ${ }^{19}$ The most important risk factor for infection was shown to be the use of immunosuppressants. ${ }^{20}$ Primary Nocardia cutaneous type can present as a superficial skin infection (cellulitis, pustules, pyoderrma), a lymphangiocutaneous infection, or a mycetoma. The latter is a deep seated progressively destructive and deforming infection. The disseminated type is usually associated with pulmonary lesions. ${ }^{20,21}$ In the disseminated type, the patient usually has a history of trauma, previous antibiotic use, or a history of local recurrence presenting with fever and purulent drainage. They are slow-growing in routine bacterial culture, rarely appearing in cultures less than 48 hours. Biopsy often reveals a dense mixed inflammatory infiltrate with suppuration, granulation tissue, fibrosis, and granuloma formation. Nocardiosis requires prolonged antibiotic treatment.$^{20}$

Another differential for non-healing wounds is fungal infection. The most important risk factor for fungal infection in immunocompromised patients, especially in cancer patients, is neutropenia. They can be either a primary cutaneous fungal infection or a dermatologic manifestation of a disseminated fungal infection.22 Our patient, while not neutropenic, was noted to have increasing oral thrush despite treatment with nystatin oral gargle. However, the increase in oral thrush or worsening fungal infection was noted only late in the course of the disease. Therefore it is unlikely to be the infectious etiology that had been causing the poor wound healing.

An algorithm in the approach of non-healing wound is presented in Figure 4. 


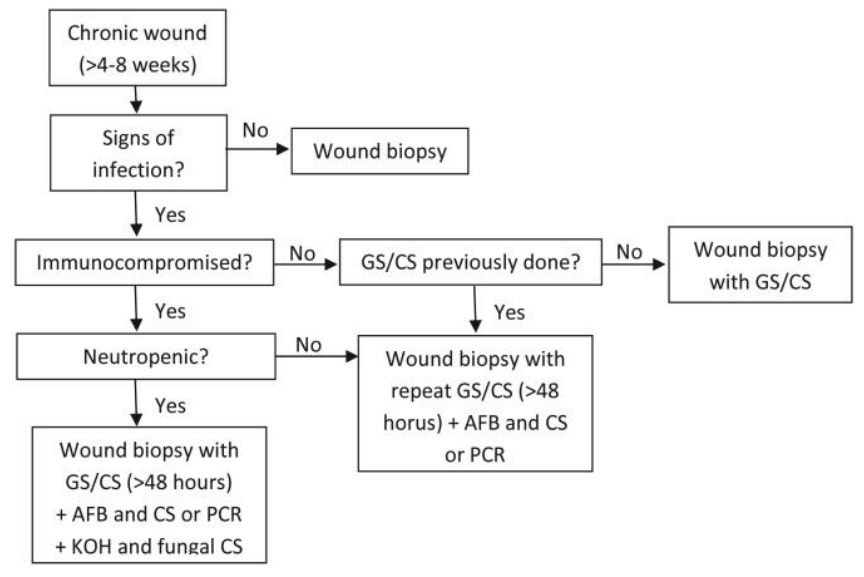

Figure 4. Proposed algorithm in the approach for patients with chronic non-healing wound.

\section{Conclusion}

In summary, we present a case of an SLE patient with a chronic non-healing wound on the right leg since 4 months prior to admission. He eventually succumbed to sepsis from nosocomial pneumonia and possible disseminated infection from the wound, or a disseminated fungal infection. The wound was shown to have suppurative and granulomatous infiltrates positive for acid fast bacilli and M. Tuberculosis on culture. Given that our patient was immunocompromised, several organisms causing chronic non-healing wound were discussed.

\section{References}

1. Van Rijswijk L, Polansky M. Predictors of time to healing deep pressure ulcers. Ostomy Wound Manage. 1994; 40(8):40-8.

2. Shah J. Approach to commonly misdiagnosed wounds and unusual leg ulcers [online]. [cited 2011 Dec]. Available from www.wounddoctors.com/chapter.doc.

3. Grey JE, Enoch S, Harding KG. ABC of wound healing: Wound assessment. BMJ. 2006; 332(7536):285-8.

4. Lopez FA, Sanders CV. Recognizing cutaneous signs of infection in immunocompromised patients. Abstr Hematol Oncol. 2003; 6(3).

5. Andrianakos AA, Tsichlis PN, Merikas EG, Marketos SG, Sharp JT, Merikas GE. Cell-mediated immunity in systemic lupus erythematosus. Clin Exp Immunol. 1977; 30(1):89-96.

6. Klein $\mathrm{NC}$, Go $\mathrm{CH}$, Cunha BA. Infections associated with steroid use. Infect Dis Clin North Am. 2001; 15(2):423-32.

7. Saraf SK, Shukla VK, Kaur P, Pandey SS. A clinico-epidemiological profile of non-healing wounds in an Indian hospital. J Wound Care. 2000; 9(5):247-50.

8. Philippine General Hospital Department of Medicine Section of Dermatology Biopsy Census for 2004-2010.

9. Gelber R. Leprosy. In: Fauci AS, Kasper DL, Longo DL, eds. Harrison's Principles of Internal Medicine, $18^{\text {th }}$ ed. U.S.A: McGraw-Hill Companies, Inc.; 2012. pp 1359-1367.

10. Scollard DM, Adams LB, Gillis TP, Krahenbuhl JL, Truman RW, Williams DL. The continuing challenges of leprosy. Clin Microbiol Rev. 2006; 19(2):338-81.

11. Schwarz R, BrandsmaW. Surgical reconstruction \& rehabilitation in leprosy and other neuropathies. $1^{\text {st }}$ Edition. 2004. Nepal. pp. 193-226.
12. Herchline T, Cunha BA, Chavis PS, et al. Tuberculosis [online]. [cited 2011 Dec]. Available from http://emedicine.medscape.com/ article/230802-overview\#showall.

13. Berger E, Batra P, Ralston J, Sanchez MR, Franks AG Jr. Atypical mycobacteria infection in an immunocompromised patient. Dermatol Online J. 2010; 16(11):21.

14. Knott L. Mycobacterial skin infections [online]. 2011 [cited 2011 Dec]. Available from http://www.patient.co.uk/doctor/Mycobacterial-SkinInfections.htm.

15. Dodiuk-Gad R, Dyachenko P, Ziv M, et al. Nontuberculous mycobacterial infections of the skin: A retrospective study of 25 cases. J Am Acad Dermatol. 2007; 57(3):413-20.

16. Scheinfeld NS, Elston DM, Tomar S, et al. Dermatologic manifestations of Mycobacterium avium-intracellulare infection [online]. 2010 [cited 2011 Dec]. Available from http://emedicine.medscape.com/ article/1105447-overview\#showall.

17. Ferreira CP, Coutinho ZF, Lourenço MC, Rocha Ada S, Martins CJ. Atypical cutaneous mycobacteriosis caused by Mycobacterium avium complex. Braz J Infect Dis. 2010; 14(3):324-6.

18. Raviglione, MC, O'Brien RJ. Tuberculosis. In: Fauci AS, Kasper DL, Longo DL, eds. Harrison's Principles of Internal Medicine, $18^{\text {th }} \mathrm{ed}$. U.S.A: McGraw-Hill Companies, Inc.; 2012. pp 1340-1359.

19. Minero MV, Marín M, Cercenado E, Rabadán PM, Bouza E, Muñoz P. Nocardiosis at the turn of the century. Medicine (Baltimore). 2009; 88(4):250-61.

20. Shook BA, Elston DM, Rapini RP, Rabadán PM, Bouza E, Muñoz P. Dermatologic manifestations of nocardiosis [online]. 2009 [cited 2011 Dec]. Available from http://emedicine.medscape.com/article/1052944overview.

21. Satterwhite TK, Wallace RJ Jr. Primary cutaneous nocardiosis JAMA. 1979; 242(4):333-6.

22. Hamed MS. Cutaneous fungal infections in immunocompromised patients. 2007. [Thesis] 